\title{
ENERGY-EFFICIENT JOINT TRANSMIT BEAMFORMING AND SUBARRAY SELECTION WITH NON-LINEAR POWER AMPLIFIER EFFICIENCY
}

\author{
Oskari Tervo*, Le-Nam Tran ${ }^{\dagger}$, and Markku Juntti* \\ ${ }^{*}$ Centre for Wireless Communications, University of Oulu, P.O.Box 4500, FI-90014, Finland \\ ${ }^{\dagger}$ Department of Electronic Engineering, Maynooth University, Maynooth, Co Kildare, Ireland \\ Email: \{oskarite,markku.juntti\}@ee.oulu.fi, lenam.tran@nuim.ie
}

\begin{abstract}
We study the problem of energy efficiency maximization (EEmax) with joint beamforming and subarray selection, by taking into account the non-linear power amplifier (PA) efficiency in a multi-user multiple-input single-output system. The subarray selection problem is formulated using the concept of perspective formulation with additional penalty term in the objective function. To tackle the resulting challenging mixed-Boolean non-convex optimization problem, we rely on continuous relaxation and successive convex approximation framework where a convex problem is solved in each iteration. Numerical results demonstrate the achieved energy efficiency gains of the subarray selection and show that non-linear PA efficiency has a significant impact on the optimization. We also observe that on contrast to using linear PA efficiency model, the non-linear PA efficiency model yields the fact that it is better to stay silent rather than transmit with very low transmit power.
\end{abstract}

Index Terms - Energy efficiency, sequential convex approximation, circuit power, mixed-Boolean programming, subarray selection.

\section{INTRODUCTION}

Future multiple-antenna technologies are facing a huge challenge due to the increasing energy consumption [1]. Bearing this in mind, recent research has been focusing on the energy-efficient transmission [2-9]. Employing more antennas requires extra circuitry, having a significant impact on the complexity and the processing power consumption. Specifically, significant power consumers are transmit power amplifiers (PAs) which are used before each of the active transmit antenna to amplify the radio frequency (RF) signal. The consumed power in each PA depends on the type and the design of the PA. As shown in many works, e.g., [10], the PA efficiency has a non-linear (concave) dependence on the output power. This means that for small output power values, the efficiency can drastically decrease for a given PA while the efficiency is larger and the sensitivity to output power variations smaller with higher output power values. Intuitively, this yields the fact that in a multi-antenna system it may be better to stay silent rather than transmit with small power.

This work was supported in part by Infotech Oulu Doctoral Program and the Academy of Finland under project Message and CSI Sharing for Cellular Interference Management with Backhaul Constraints (MESIC) belonging to the WiFIUS program with NSF. It was also supported by a research grant from Science Foundation Ireland and is co-funded by the European Regional Development Fund under Grant 13/RC/2077. Further supporters have been KAUTE foundation, HPY Research foundation, Walter Ahlström Foundation, and Tauno Tönning Foundation.
Another important fact arising from the use of large numbers of antennas is the limited numbers of RF chains, i.e., the latter may be smaller than the former. This means that the beamforming gain and the number of parallel streams becomes limited. However, huge numbers of antenna elements can be installed in the transmitter in particular at higher center frequencies, like mm-waves [11]. The same has also been proposed for more conventional microwave frequencies below $10 \mathrm{GHz}$ [11]. One option to benefit from the large number of antenna elements with a smaller number of transceiver processing chains to achieve array gain is to divide the antennas into subarrays each of which is connected to an RF chain. In general, the number of RF chains may be smaller than that of subarrays. This kind of system model is called hybrid beamforming [12] which becomes very relevant in mm-wave systems. In addition to enable hybrid beamforming, the model results in smaller power variations between antennas, which means that the non-linear effects of the power amplifiers become less significant. Such antenna grouping could be used even in the case with conventional antenna selection problem, where we could group the antennas and select the sub-groups just to simplify the antenna selection problem.

In this paper, we consider the energy efficiency with joint beamforming and subarray selection (JBSAS) and non-linear efficiency of PA in multiuser multiple-input single-output (MISO) system. This is different and more practical system compared to the conventional joint beamforming and antenna selection problem considered in [9] where each antenna is individually connected with an RF chain (using a switch). In addition, here we consider non-linear PA efficiency whereas the linear case has been assumed in the prior works. To tackle the resulting challenging mixed-Boolean non-convex optimization problem, we rely on continuous relaxation and successive convex approximation framework where a convex problem is solved in each iteration. Numerical results demonstrate the achieved energy efficiency gains of the subarray selection and shows that non-linear PA efficiency has a significant impact on the optimization. We also observe that on contrast to using linear PA efficiency model, the nonlinear PA efficiency model yields the fact that it is better to stay silent rather than transmit with very low transmit power.

Notations: $\mathbf{X}^{T}$ is transpose of $\mathbf{X} ;|x|$ represents the absolute value of $x$ and $\|\mathbf{x}\|_{2}$ is $\ell_{2}$-norm of $\mathbf{x} ; \operatorname{Im}(\mathbf{X})$ means the imaginary part of $\mathbf{X}$.

\section{PROBLEM FORMULATION}

\subsection{System Model}

We consider a single-cell MISO downlink channel, where a base station with $N$ antenna elements transmits data to $K$ single-antenna 
receivers. ${ }^{1}$ The antenna elements are divided into $G$ groups with $N_{g}$ antennas in each group; the antenna elements belonging to a group form a subarray. The set of groups and the set of antennas in group $g$ is denoted as $\mathcal{G}$ and $\mathcal{N}_{g}$, respectively. Each antenna group is connected to a single RF chain and each antenna has its own power amplifier. (The number of RF chains can be smaller than the number of subarrays which means that some RF chains can be connected to many subarrays by using a switch.) The channel (row) vector from the BS to user $k$ is represented by $\mathbf{h}_{k} \in \mathbb{C}^{1 \times N}$. We adopt linear transmit beamforming, where data symbol $s_{k}$ intended for user $k$ is multiplied with the beamformer $\mathbf{w}_{k} \in \mathcal{C}^{N \times 1}$ before being transmitted. For each antenna group $g$, we use group specific beamformer so that the beamformer coefficients towards user $k$ are $\mathbf{m}_{g, k} \triangleq$ $\left[\mathbf{w}_{k}\right]_{i} \mathbf{1}_{N_{g}} \in \mathcal{C}^{N_{g} \times 1}$ where $\left[\mathbf{w}_{k}\right]_{i}\left(i \in \mathcal{N}_{g}\right)$ is the beamformer coefficient from the $i$ th transmit antenna to user $k^{2}$. As a result, the total beamformer towards user $k$ is $\mathbf{w}_{k} \triangleq\left[\mathbf{m}_{1, k}^{T}, \mathbf{m}_{2, k}^{T}, \ldots, \mathbf{m}_{G, k}^{T}\right]^{T}$. Accordingly, the received signal at the $k$ th user is given by

$$
y_{k}=\mathbf{h}_{k} \mathbf{w}_{k} s_{k}+\sum_{\substack{j \in \mathcal{K} \\ j \neq k}} \mathbf{h}_{k} \mathbf{w}_{j} s_{j}+n_{k}
$$

where $\mathcal{K} \triangleq\{1, \ldots, K\}$ and $n_{k}$ is the background noise with distribution assumed to be $\mathcal{C N}\left(0, N_{0}\right)$. The data streams are independent and have zero mean and unit power. It is also assumed that both the users and BSs have perfect channel state information. Since the users are not allowed to cooperate, the multi-user interference is treated as Gaussian noise and the data rate of user $k$ is given by

$$
R_{k}(\mathbf{w})=\log \left(1+\gamma_{k}(\mathbf{w})\right)
$$

where $\mathbf{w} \triangleq\left[\mathbf{w}_{1}^{T}, \mathbf{w}_{2}^{T}, \ldots, \mathbf{w}_{K}^{T}\right]^{T}$ and SINR at user $k$ is

$$
\gamma_{k}(\mathbf{w}) \triangleq \frac{\left|\mathbf{h}_{k} \mathbf{w}_{k}\right|^{2}}{N_{0}+\sum_{\substack{j \in \mathcal{K} \\ j \neq k}}\left|\mathbf{h}_{k} \mathbf{w}_{j}\right|^{2}}
$$

\subsection{Power Consumption Model}

Here we consider class B type power amplifier model as in [10], where the output power dependent power consumption of the $i$ th power amplifier is modeled as

$$
p_{i, \text { data }}\left(p_{i, \text { out }}\right) \triangleq c \sqrt{P_{\max }} \sqrt{p_{i, \text { out }}},
$$

where $c$ is a constant which tunes the efficiency slope, $p_{i \text {,out }}$ is the output power of PA $i$, and $P_{\max }$ is the maximum output power allowed to a specific power amplifier. As a result, the total power consumption of the transmission can be modeled as

$$
P_{\mathrm{tot}}=\sum_{i \in \mathcal{N}} p_{i, \text { data }}+\sum_{g \in \mathcal{G}} a_{g} P_{\mathrm{RF}}+P_{0}
$$

where $P_{\mathrm{RF}}$ is the power consumed in each active RF chain, $a_{g} \in$ $\{0,1\}$ is the antenna group selection variable of group $g$, i.e., $a_{g}=1$ if group $g$ is selected for transmission, and $P_{0}$ is the data independent total fixed circuit power consumed in transmitter.

\subsection{Energy Efficiency Maximization}

To model the antenna group specific output power, we collect the beamforming weights of all the users associated with $g$ th antenna group into a vector denoted by $\overline{\mathbf{w}}_{g} \triangleq\left[\mathbf{m}_{g, 1}^{T}, \mathbf{m}_{g, 2}^{T}, \cdots, \mathbf{m}_{g, K}^{T}\right]^{T} \in$ $\mathbb{C}^{N_{g} K \times 1}$. Then, we must ensure $a_{g}=0 \Rightarrow \overline{\mathbf{w}}_{g}=0$, i.e., the beamforming weights associated with the $g$ th antenna group are forced

\footnotetext{
${ }^{1}$ The mathematical presentations can be straightforwardly extended to multi-cell MISO systems with centralized algorithms.

${ }^{2}$ This means that the signal is equally divided into antennas inside a group.
}

to zero as $a_{g}=0$. This condition is satisfied with the constraint $\left\|\overline{\mathbf{w}}_{g}\right\|_{2}^{2} \leq a_{g} v_{g}$, where $v_{g}$ can be interpreted as a soft power level for the $g$ th antenna group and is optimized under maximum power $P_{g}[13,14]$. On the other hand, for the PA power consumption we need to define antenna specific output power. To this end, let us collect the beamforming weights related to antenna $i$ to a vector $\hat{\mathbf{w}}_{i} \triangleq\left[\left[\mathbf{w}_{1}\right]_{i},\left[\mathbf{w}_{2}\right]_{i}, \cdots,\left[\mathbf{w}_{K}\right]_{i}\right]^{T} \in \mathbb{C}^{N \times 1}$. Because the signal power is equally divided to the antennas inside the group, it is relevant to use the group specific power constraints. In the case of multiantenna design, we can write the output power dependent power consumption as $c_{i} \sqrt{P_{\max }}|| \hat{\mathbf{w}}_{i} \|_{2}$. By defining $u_{i}=c_{i} \sqrt{P_{\max }}$ and by plugging the efficiency expression into the problem, we can formulate the problem of energy efficiency maximization with per-user SINR constraints, antenna group specific power constraints as

$$
\begin{array}{ll}
\max _{\mathbf{w}, \mathbf{v}, \mathbf{a}} & \frac{\sum_{k \in \mathcal{K}} R_{k}(\mathbf{w})}{\sum_{i \in \mathcal{N}} u_{i}\left\|\hat{\mathbf{w}}_{i}\right\|_{2}+\sum_{g \in \mathcal{G}} a_{g} P_{\mathrm{RF}}+P_{0}} \\
\text { s.t. } & \gamma_{k} \geq \bar{\gamma}_{k}, \forall k \in \mathcal{K} \\
& \left\|\overline{\mathbf{w}}_{g}\right\|_{2}^{2} \leq a_{g} v_{g}, \forall g \in \mathcal{G} \\
& v_{g} \leq P_{g}, \forall g \in \mathcal{G} \\
& \sum_{g \in \mathcal{G}} a_{g} \leq L \\
& a_{g} \in\{0,1\}, \forall g \in \mathcal{G}
\end{array}
$$

where $\mathbf{v} \triangleq\left[v_{1}, \ldots, v_{G}\right]^{T}, \mathbf{a} \triangleq\left[a_{1}, \ldots, a_{G}\right]^{T}$, and $L$ is the maximum number of RF chains. We can see that compared to the conventional design, where the efficiency is assumed to be fixed [9], PA consumption of each antenna scales with $\left\|\hat{\mathbf{w}}_{i}\right\|_{2}$ instead of $\left\|\hat{\mathbf{w}}_{i}\right\|_{2}^{2}$. This suggests that for small transmit powers, the total power consumption is larger than in the conventional design, since the PA efficiency is poor. However, for larger power values it is the opposite.

\section{OPTIMIZATION ALGORITHM}

Problem (6) is a mixed-Boolean nonconvex fractional program for which finding an optimal solution may end up with an exhaustive search. Also, even if some of $a_{g}$ 's are fixed to 0 or 1 and the others are relaxed to be continuous over the interval $[0,1]$, the resulting problem is still nonconvex. Thus, it is practical to rely on suboptimal methods to solve the problem. Here the aim is to provide lowcomplexity solution by the combination of continuous relaxation and successive convex approximation. The problem structure resembles the JBAS-EEmax problem in [9] with minor differences. Motivated by this, we use similar ideas to transform the problem to a more easily tractable form.

As a first step, we relax the binary variables so that they can get continuous values in the interval [0,1], i.e., the constraint (6f) is replaced by $0 \leq a_{g} \leq 1$. After the relaxation, we can observe that all the constraints in (6) are convex and the difficulty is in the objective function (6a) by noting the fact that the constraint in (6b) is equivalent to the following SOC representation [15]

$$
\frac{1}{\sqrt{\gamma_{k}}} \mathbf{h}_{k} \mathbf{w}_{k} \geq\left(N_{0}+\sum_{\substack{j \in \mathcal{K} \\ j \neq k}}\left|\mathbf{h}_{k} \mathbf{w}_{j}\right|^{2}\right)^{\frac{1}{2}}, \operatorname{Im}\left(\mathbf{h}_{k} \mathbf{w}_{k}\right)=0 .
$$

In [9], we have derived the SCA based method for the problem with the same structure as (6). However, as noted in [9], the SCA with continuous relaxation often yields solutions where all the $a_{g}$ 's are close to zero which makes the mapping to binary values difficult. Motivated by this, we first equivalently transform problem (6) as [9] 


$$
\begin{array}{ll}
\max & t \\
\text { s.t. } & \sum_{k \in \mathcal{K}} \log \left(1+\gamma_{k}\right) \geq \sqrt{t z} \\
& \sqrt{z} \geq \sum_{i \in \mathcal{N}} u_{i}\left\|\hat{\mathbf{w}}_{i}\right\|_{2}+\sum_{g \in \mathcal{N}_{g}} a_{g} P_{\mathrm{RF}}+P_{0} \\
& 0 \leq a_{g} \leq 1, \forall g \in \mathcal{G} \\
& \text { (6c), (7), (6d), (6e), }
\end{array}
$$

where the variables are $\mathbf{w}, \mathbf{v}, \mathbf{a}$ and newly introduced variables $t$ and $z$ denote the squared energy efficiency and squared power consumption, respectively. The equivalence between (8) and (6) is because (8b) and (8c) must hold with equality at the optimum, and that maximizing $\sqrt{t}$ equals to maximizing $t$. Then, to make mapping of binary variables easier, we consider the following problem

$$
\begin{array}{ll}
\max & t-\rho \sum_{g \in \mathcal{G}} v_{g} \\
\text { s.t. } & \sum_{k \in \mathcal{K}} \log \left(1+\gamma_{k}\right) \geq \sqrt{t z} \\
& \sqrt{z} \geq \sum_{i \in \mathcal{N}} u_{i}\left\|\hat{\mathbf{w}}_{i}\right\|_{2}+\sum_{g \in \mathcal{N}_{g}} a_{g} P_{\mathrm{RF}}+P_{0} \\
& 0 \leq a_{g} \leq 1, \forall g \in \mathcal{G} \\
& (6 \mathrm{c}),(7),(6 \mathrm{~d}),(6 \mathrm{e}),
\end{array}
$$

where we have added a penalty term $\rho \sum_{g \in \mathcal{G}} v_{g}(\rho \geq 0)$ to the objective. Intuitively, without that, (6c) tends to make $a_{g}$ small and $v_{g}$ large. However, when we penalize the objective with $v_{g}$, the problem tends to make $v_{g}$ smaller and $a_{g}$ either smaller or larger (likely, small $a_{g}$ 's go towards zero). Next we derive the solution for the above problem.

Although (9c) is convex as such, we note that it can be equivalently expressed as

$$
(9 \mathrm{c}) \Leftrightarrow\left\{\begin{array}{l}
\frac{z+1}{2} \geq\left\|\left[\frac{z-1}{2}, z^{\prime}\right]^{T}\right\|_{2} \\
z^{\prime}-\sum_{g \in \mathcal{G}} a_{g} P_{\mathrm{RF}}-P_{0} \geq \sum_{i \in \mathcal{N}} u_{i} \xi_{i} \\
\left\|\hat{\mathbf{w}}_{i}\right\|_{2} \leq \xi_{i}, i \in \mathcal{N}
\end{array}\right.
$$

where $z^{\prime}$ and $\boldsymbol{\xi} \triangleq\left[\xi_{1}, \xi_{2}, \ldots, \xi_{N}\right]^{T}$ are new optimization variables. The above expressions become the second-order cone (SOC) constraints if binary variables are relaxed to be continuous. To further expose the hidden convexity, we express (9b) as

$$
\begin{aligned}
\sum_{k \in \mathcal{K}} \log \vartheta_{k} & \geq \sqrt{t z} \\
1+\gamma_{k} & \geq \vartheta_{k}, \forall k \in \mathcal{K}
\end{aligned}
$$

where we have introduced new variables $\vartheta \triangleq\left[\vartheta_{1}, \vartheta_{2}, \ldots, \vartheta_{K}\right]^{T}$. Note that (11a) can be equivalently replaced by the constraints

$$
\begin{aligned}
\sum_{k \in \mathcal{K}} \alpha_{k} & \geq \sqrt{t z} \\
\log \vartheta_{k} & \geq \alpha_{k} \Longleftrightarrow \vartheta_{k} \geq e^{\alpha_{k}}, \forall k \in \mathcal{K}
\end{aligned}
$$

with new variables $\boldsymbol{\alpha} \triangleq\left[\alpha_{1}, \alpha_{2}, \ldots, \alpha_{K}\right]^{T}$ representing the rate of each user $k$. From (11) and (12) we can equivalently rewrite (9b) as the set of following constraints

$$
\left\{\begin{array}{l}
1+\gamma_{k} \geq \vartheta_{k}, \forall k \in \mathcal{K} \\
\vartheta_{k} \geq e^{\alpha_{k}}, \forall k \in \mathcal{K} \\
\sum_{k \in \mathcal{K}} \alpha_{k} \geq \sqrt{t z}
\end{array}\right.
$$

Next, motivated by [16], we replace the constraint $1+\gamma_{k} \geq \vartheta_{k}$ in (13a) by new inequality constraints as follows

$$
\mathbf{h}_{k} \mathbf{w}_{k} \geq \sqrt{\left(\vartheta_{k}-1\right) \beta_{k}}, \forall k \in \mathcal{K}
$$

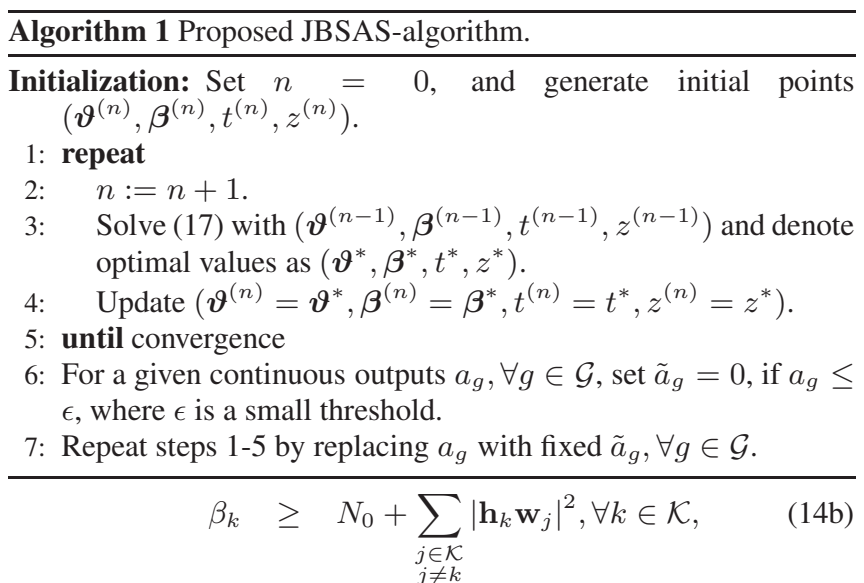

where (14b) are convex with new variables $\boldsymbol{\beta} \triangleq\left[\beta_{1}, \beta_{2}, \ldots, \beta_{K}\right]^{T}$ and can be also expressed as SOC constraints. Note that $\beta_{k}$ can be interpreted as inter-user interference plus noise experienced by user $k$. We now arrive at an equivalent reformulation of (9) given by

$$
\begin{array}{ll}
\max & t-\rho \sum_{g \in \mathcal{G}} v_{g} \\
\text { s.t. } & \mathbf{h}_{k} \mathbf{w}_{k} \geq \sqrt{\left(\vartheta_{k}-1\right) \beta_{k}}, \forall k \in \mathcal{K} \\
& \sum_{k \in \mathcal{K}} \alpha_{k} \geq \sqrt{t z} \\
& (6 \mathrm{c}),(7),(9 \mathrm{c}),(13 \mathrm{~b}),(14 \mathrm{~b}),(9 \mathrm{~d}),(6 \mathrm{e}),(6 \mathrm{~d})
\end{array}
$$

where the optimization variables are $t, z, \mathbf{w}, \mathbf{a}, \mathbf{v}, \boldsymbol{\vartheta}, \boldsymbol{\alpha}, \boldsymbol{\beta}$. We remark that all the constraints listed in (15d) are convex if the binary variables are continuous, and the constraints (15b) and (15c) are in a form where the left side is linear and the right side is in a form of geometric mean function which is jointly concave. Here, we propose an SCA based beamforming design, which approximates (15) by a convex formulation in each iteration. Let $x^{(n)}$ be the value of the optimization variable $x$ after the $n$th step of the iterative procedure. Since (15b) and (15c) are of the same type, we present the iterative approximation for (15c), then that for (15b) follows in a similar manner. First, we note that the function $\sqrt{t z}$ is jointly concave with respect to $t$ and $z$ on the domain $t \geq 0, z \geq 0$. Thus, a convex upper approximation of the right side of (15c) can be found as

$$
\begin{aligned}
\sqrt{t z} \leq \sqrt{t^{(n)} z^{(n)}}+\frac{1}{2} & \left(\frac{z^{(n)}}{t^{(n)}}\right)^{1 / 2}\left(t-t^{(n)}\right) \\
& +\frac{1}{2}\left(\frac{t^{(n)}}{z^{(n)}}\right)^{1 / 2}\left(z-z^{(n)}\right) .
\end{aligned}
$$

In fact, (16) is the first order of the function $\sqrt{t z}$ around the point $\left(t^{(n)}, z^{(n)}\right)$. We note that in our formulation $z$ and $t$ are strictly positive, and, thus, the first order approximation in (16) is well defined over the whole feasible set of (15). This argument also applies to the first order approximation of $\sqrt{\left(\vartheta_{k}-1\right) \beta_{k}}$. Based on these, we can approximate (15) at iteration $n$ by the following convex program

$$
\begin{array}{ll}
\max & t-\rho \sum_{g \in \mathcal{G}} v_{g} \\
\text { s.t. } & \mathbf{h}_{k} \mathbf{w}_{k} \geq L A^{(n)}\left(\vartheta_{k}, \beta_{k}\right), \forall k \in \mathcal{K} \\
& \sum_{k \in \mathcal{K}} \alpha_{k} \geq L A^{(n)}(t, z) \\
& (6 \mathrm{c}),(7),(9 \mathrm{c}),(13 \mathrm{~b}),(14 \mathrm{~b}),(9 \mathrm{~d}),(6 \mathrm{e}),(6 \mathrm{~d})
\end{array}
$$

where $L A^{(n)}(\cdot)$ stands for the first order approximation of the arguments, i.e., the right side of the inequality (16) for (17c). Note that the feasible set of (17) is also feasible to (15). 


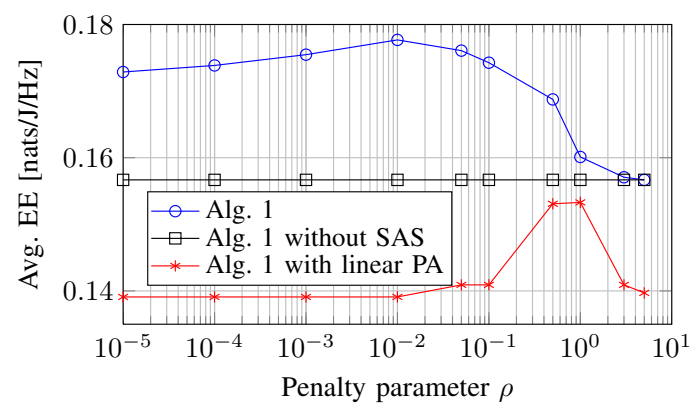

Fig. 1: The effect of penalty parameter with $P_{\mathrm{RF}}=1 \mathrm{~W}$.

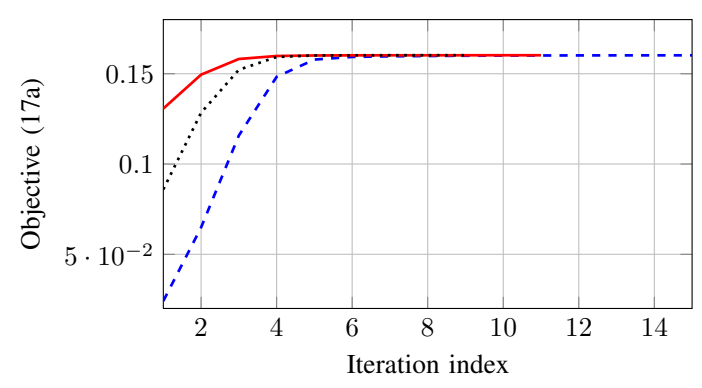

Fig. 2: Convergence of Alg. 1 (steps 1-5) with $P_{\mathrm{RF}}=1 \mathrm{~W}$.

We note that due to the exponential cone in (13b), problem (17) as such is categorized as a generalized nonlinear convex program which can be solved by available nonlinear solvers such as Fmincon. However, as presented in [9], (13b) can be approximated as a system of SOC constraints. By using the approximation, (17) becomes SOCP which can be solved using efficient SOCP solvers such as MOSEK, ECOS or GUROBI. The convergence of the objective function can be shown as in [9]. After solving the relaxed problem using the algorithm above, we simply switch off all the antenna groups which have $a_{g} \leq \epsilon$, where $\epsilon$ is a small threshold, and calculate the beamformers applying the same algorithm with reduced dimensions for this fixed sets of subarrays.

\section{NUMERICAL RESULTS}

We consider a base station with $N=27$ antennas, divided into $G=9$ groups of $N_{g}=3$ antennas. The number of users is $K=3$ and the channels are Rayleigh fading. We set $P_{g}=3 \mathrm{~W}, \forall g \in$ $\mathcal{G}, N_{0}=1, L=G, \bar{\gamma}_{k}=0.1, \forall k \in \mathcal{K}, P_{0}=1 \mathrm{~W}$ and PA efficiency parameters as $c_{i}=c=4.7$ [10] and $P_{\max }=P_{g} / 3=1 \mathrm{~W}$. The proposed method is compared to Alg. 1 without subarray selection, and Alg. 1 with linear PA efficiency. In case of linear PA, the PA power consumption is a linear function of output power, i.e., we set $u_{i}=c_{i},\left\|\hat{\mathbf{w}}_{i}\right\|_{2}$ in (9c) is replaced with $\left\|\hat{\mathbf{w}}_{i}\right\|_{2}^{2}$, and after solving the JBSAS problem, the energy efficiency is calculated by assuming the non-linear efficiency model.

In Fig. 1, we show the effect of penalty parameter $\rho$ on the average energy efficiency. We can see that the achieved energy efficiency gain (because of subarray selection) depends on $\rho$. In the considered setup, $\rho=0.01$ gives the best result for the proposed algorithm and $\rho=3$ for the method with linear PA. Thus, we use those values for the rest of the figures. It can be observed that Alg. 1 with linear PA efficiency gives significantly worse performance than even Alg. 1 without SAS. This is because when all the power amplifier efficiencies are approximated wrongly, the sum effect becomes huge when

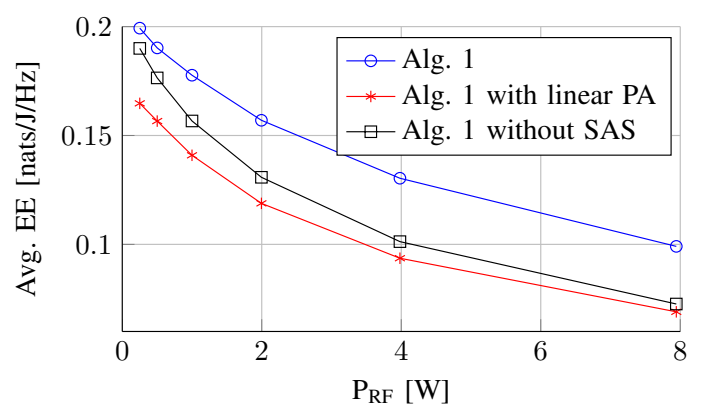

Fig. 3: Energy efficiency versus $P_{\mathrm{RF}}$.

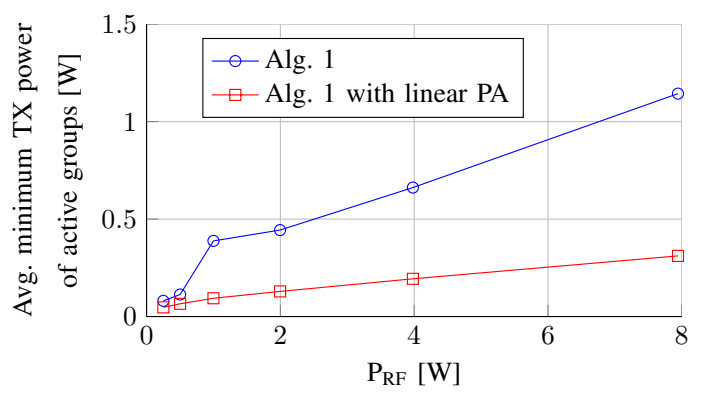

Fig. 4: Average minimum TX power of active groups versus $P_{\mathrm{RF}}$.

solving the JBSAS problem.

Fig. 2 illustrates the convergence of the objective function (17a) for three different initial points when solving the relaxed problem. We can see that the convergence rate is fast in the considered settings and the objective value after convergence is the same when starting from different initial points.

Fig. 3 compares the average energy efficiency versus RF chain specific power $P_{\mathrm{RF}}$. We can see the gains achieved by switching off some of the RF chains. It can be seen that if PA power consumption is assumed to linear function of output power, there is a remarkable difference in the achieved energy efficiency. This is mainly because in case of linear efficiency, it is energy-efficient to transmit even with very small transmit powers, which means that we still keep more RF chains active. On the other hand, in practice, the PA efficiency is very poor at low output powers, which means that it is better to keep the antenna silent. This is further illustrated in the next figure.

Fig. 4 shows the minimum transmit power per active group versus $P_{\mathrm{RF}}$. This verifies that with linear model, we tend to transmit with low transmit powers, yielding the fact that we keep more subarrays active. Thus, it is important to consider the non-linear model because in practice the efficiency is very poor at low output power levels so it is better to switch of the subarray rather than keep it active with small transmit power.

\section{CONCLUSION}

This paper studied energy-efficient joint beamforming and subarray selection in multiuser MISO systems. The resulting mixed-Boolean nonconvex optimization problem was solved using the combination of continuous relaxation and successive convex approximation with additional penalty parameter in the objective. Numerical results illustrated the achieved energy efficiency gains of the subarray selection and shows that non-linear PA efficiency has a significant impact on the optimization. 


\section{REFERENCES}

[1] D. Feng, C. Jiang, G. Lim, J. Cimini, L.J., G. Feng, and G. Li, "A survey of energy-efficient wireless communications," IEEE Commun. Surveys Tuts., vol. 15, no. 1, pp. 167-178, First 2013.

[2] C. Isheden, Z. Chong, E. Jorswieck, and G. Fettweis, "Framework for link-level energy efficiency optimization with informed transmitter," IEEE Trans. Wireless Commun., vol. 11, no. 8, pp. 2946-2957, Aug. 2012.

[3] D. Ng, E. Lo, and R. Schober, "Energy-efficient resource allocation in OFDMA systems with large numbers of base station antennas," IEEE Trans. Wireless Commun., vol. 11, no. 9, pp. 3292-3304, Sep. 2012.

[4] L. Venturino, A. Zappone, C. Risi, and S. Buzzi, "Energyefficient scheduling and power allocation in downlink OFDMA networks with base station coordination," IEEE Trans. Wireless Commun., vol. 14, no. 1, pp. 1-14, Jan 2015.

[5] T. Wang and L. Vandendorpe, "On the optimum energy efficiency for flat-fading channels with rate-dependent circuit power," IEEE Trans. Commun., vol. 61, no. 12, pp. 4910-4921, December 2013.

[6] Y. Li, Y. Tian, and C. Yang, "Energy-efficient coordinated beamforming with individual data rate constraints," in Personal Indoor and Mobile Radio Communications (PIMRC), 2013 IEEE 24th International Symposium on, Sept 2013, pp. 1040-1044.

[7] C. Jiang and L. Cimini, "Downlink energy-efficient multiuser beamforming with individual SINR constraints," in IEEE MILCOM, 2011, pp. 495-500.

[8] O. Tervo, A. Tölli, M. Juntti, and L. N. Tran, "Energyefficient coordinated beamforming with rate dependent processing power," in Proc. IEEE Int. Workshop Signal Process. Adv. Wireless Commun., July 2016, pp. 1-5.

[9] O. Tervo, L.-N. Tran, and M. Juntti, "Optimal energy-efficient transmit beamforming for multi-user MISO downlink," IEEE Trans. Signal Process., vol. 63, no. 20, pp. 5574-5588, Oct 2015.

[10] X. Shi, W. Xu, X. Zhou, and J. Lin, "Energy efficiency optimization in OFDM-based cognitive radio systems: Impact of power amplifiers," in IEEE 81st Veh. Tech. Conf., May 2015, pp. 1-5.

[11] T. E. Bogale and L. B. Le, "Massive MIMO and mmwave for 5G wireless hetnet: Potential benefits and challenges," IEEE Veh. Technol. Mag., vol. 11, no. 1, pp. 64-75, March 2016.

[12] F. Sohrabi and W. Yu, "Hybrid Digital and Analog Beamforming Design for Large-Scale Antenna Arrays," IEEE J. Sel. Topics Signal Process., vol. 10, pp. 501-513, Apr. 2016.

[13] O. Günlük and J. Linderoth, "Perspective reformulations of mixed integer nonlinear programs with indicator variables," Mathematical Programming, vol. 124, no. 1-2, pp. 183-205, 2010. [Online]. Available: http://dx.doi.org/10.1007/s10107010-0360-z

[14] P. Bonami, M. Kilinç, and J. Linderoth, "Algorithms and software for convex mixed integer nonlinear programs," in Mixed Integer Nonlinear Programming, ser. The IMA Volumes in Mathematics and its Applications, J. Lee and S. Leyffer, Eds. Springer New York, 2012, vol. 154, pp. 1-39. [Online]. Available: http://dx.doi.org/10.1007/978-1-4614-1927-3_1
[15] A. Wiesel, Y. Eldar, and S. Shamai, "Linear precoding via conic optimization for fixed MIMO receivers," IEEE Trans. Signal Process., vol. 54, no. 1, pp. 161-176, Jan. 2006.

[16] L.-N. Tran, M. F. Hanif, A. Tölli, and M. Juntti, "Fast converging algorithm for weighted sum rate maximization in multicell MISO downlink," IEEE Signal Process. Lett., vol. 19, no. 12, pp. 872-875, Dec. 2012. 